CERN-TH/2001-323

SINP/TNP/01-27

hep-ph/0111345

\title{
Testing orbifold models of Supersymmetric Grand Unification
}

\author{
Gautam Bhattacharyya ${ }^{1 *}$ and K. Sridhar ${ }^{2 \dagger} \ddagger$ \\ 1. Saha Institute of Nuclear Physics, 1/AF Bidhan Nagar, Kolkata 700064, India \\ 2. Theory Division, CERN, CH-1211, Geneva 23, Switzerland
}

\begin{abstract}
In a model of supersymmetric SU(5) Grand Unification with a spatial dimension described by the orbifold $S^{1} /\left(Z_{2} \times Z_{2}^{\prime}\right)$, proton decay is naturally suppressed at all orders. This is achieved by a suitable implementation of the discrete symmetries on the brane. But baryon number violating interactions are present in this model. We propose a few possible experimental tests of this model which exploit the effect of the baryon number violating couplings on low-energy observables like neutron-antineutron oscillations, double nucleon decay into two kaons, hadronic decay widths of the $Z$ boson, and $t \bar{t}$ production cross-section in Run II of the Tevatron.
\end{abstract}

*gb@theory.saha.ernet.in

${ }^{\dagger}$ sridhar@theory.tifr.res.in

${ }^{\ddagger}$ On leave of absence from the Tata Institute of Fundamental Research, Homi Bhabha Road, Mumbai 400 005, India. 
Weak scale Supersymmetry (SUSY) is arguably the most promising candidate for describing physics beyond the Standard Model (SM). It was introduced in the first place to protect the electroweak scale from destabilizing divergences [1]. Later, the idea of SUSY being embedded into some Grand Unified Theories (GUT) [2] received a significant boost when it was observed that the Minimal Supersymmetric Standard Model (MSSM) has the right particle content to ensure that the gauge couplings indeed meet a high scale $M_{\mathrm{GUT}} \sim 2 \times 10^{16} \mathrm{GeV}$ [3]. The unification condition, in fact, predicts the existence of superparticles in the $\mathrm{TeV}$ range which suggests that they may well be discovered in collider experiments in the near future.

While the SUSY GUT models are theoretically very appealing, they face stiff experimental challenges. For instance, the most minimal version of the supersymmetric $S U(5)$ model has now been excluded [4] by the Super-Kamiokande lower limit on the proton lifetime in the $p \rightarrow K^{+} \bar{\nu}$ channel $\left(6.7 \times 10^{32}\right.$ years at $90 \%$ C.L. [5]). The argument goes as follows: Requiring that the gauge coupling constants exactly unify at the GUT scale, a precise knowledge of the strong coupling constant $\alpha_{S}\left(M_{Z}\right)$ from LEP has now sharpened the interval $3.5 \times 10^{14} \leq M_{H_{C}} \leq 3.6 \times 10^{15}$ (in GeV) at 90\% C.L., where $M_{H_{C}}$ is the mass of the coloured triplet Higgs boson that mediates the dominant decay of the proton. On the other hand, the new Super-Kamiokande limit mentioned above imposes the constraint: $M_{H_{C}} \geq 7.6 \times 10^{16} \mathrm{GeV}$. Such a gross disagreement between these two limits excludes minimal supersymmetric SU(5) even in the decoupling limit. To save the GUT models from this embarassment, one can either look for suppressing the dimension-5 proton decay operator in some way, or attempt to prepare a natural ground to solve the doublet-triplet splitting problem by pushing the coloured triplet Higgs mass very heavy while keeping the doublet Higgs at the weak scale.

In recent years, there has been a great resurgence of interest in the physics of extra dimensions. More recently, the implications of models in which SUSY is embedded into GUT in higher dimensions, and both SUSY and GUT breaking being eventually realised by orbifold compactifications, have been explored. There are several models in the literature $[6,7,8]$, but one recent suggestion, originally due to Kawamura [6] and developed, in particular, by Altarelli and Feruglio [7], is particularly interesting because it provides a framework for an explicit realisation of minimal SUSY embedded into SU(5) GUT while avoiding all the aforementioned pitfalls of conventional SUSY GUT scenarios. We refer to this model as the Kawamura-Altarelli-Feruglio (KAF) model. We would like to point out at the very outset, that the model of Altarelli and Feruglio [7] does differ in the details of the interaction of the brane and bulk fields from the original Kawamura framework [6] and in all such cases of difference what we refer to as the KAF model is only the Altarelli-Feruglio model.

To recapitulate the basics, the minimal fermionic representation of the 5-dimensional Lorentz group is a 4-component spinor, which decomposes under the 4-dimensional Lorentz group as two 2-component Weyl spinors. Thus in 5 dimensions the minimal SUSY has 8 real supercharges which corresponds to $N=(1,1)$ or equivalently $N=2$ supersymmetry. In other words, a hypermultiplet $\left(\Phi\left(x^{\mu}, y\right), \Phi^{c}\left(x^{\mu}, y\right)\right)$ in 5 dimensions corresponds to two sets of degenerate $N=1$ chiral multiplets $\Phi\left(x^{\mu}, y\right)$ and $\Phi^{c}\left(x^{\mu}, y\right)$ in 4-dimensional language with the 5th $(y)$ coordinate acting as abel on the 4-dimensional fields. The 5-dimensional theory is necessarily a vector-like theory with $\Phi$ and $\Phi^{c}$ transforming under $\mathrm{SU}(5)$ as 5 and $\overline{5}$, respectively. Also, in 5 dimensions the only interaction is the gauge interaction. In the KAF model, one starts with a 5-dimensional GUT with minimal SU(5) gauge group and $N=2$ SUSY. The 5-dimensional spacetime is factorised into a product of the four dimensional spacetime $M^{4}$ (labelled by the co-ordinates $x^{\mu}$ ) with the extra spatial dimension compactified on the orbifold $S^{1} /\left(Z_{2} \times Z_{2}^{\prime}\right)$ (labelled by the co-ordinate $\left.y=x_{5}\right)$ ). The radius, $R$, of the circle $S^{1}$ is chosen to be of the order of $M_{\mathrm{GUT}}^{-1}$. The orbifold construction is as follows: One starts by 
dividing $S^{1}$ by a $Z_{2}$ transformation $y \rightarrow-y$ and then a further division by $Z_{2}^{\prime}$ which acts as $y^{\prime} \rightarrow-y^{\prime}$ with $y^{\prime}=y+\pi R / 2$. After these identifications, the spacetime is the interval $[0, \pi R / 2]$ with a brane located at each fixed point $y=0$ and $y=\pi R / 2$. As a result of the two reflections, the branes at $y=\pi R$ and $-\pi R / 2$ are identified with those at $y=0$ and $y=\pi R / 2$, respectively. The reason for the action of two discrete symmetries $Z_{2}$ and $Z_{2}^{\prime}$ becomes apparent when the following considerations are taken into account. Let us consider a generic field $\phi\left(x^{\mu}, y\right)$ exisiting in the 5-dimensional bulk. The $Z_{2}$ and $Z_{2}^{\prime}$ parities (called $P$ and $P^{\prime}$, respectively) are defined for this field as

$$
\begin{aligned}
\phi\left(x^{\mu}, y\right) & \rightarrow \phi\left(x^{\mu},-y\right)=P \phi\left(x^{\mu}, y\right) \\
\phi\left(x^{\mu}, y^{\prime}\right) & \rightarrow \phi\left(x^{\mu},-y^{\prime}\right)=P^{\prime} \phi\left(x^{\mu}, y^{\prime}\right) .
\end{aligned}
$$

Using the notation $\phi_{ \pm \pm}$for the fields with $\left(P, P^{\prime}\right)=( \pm, \pm)$, a Fourier expansion in $y$ yields:

$$
\begin{aligned}
\phi_{++}\left(x^{\mu}, y\right) & =\sqrt{\frac{2}{\pi R}} \sum_{n=0}^{\infty} \phi_{++}^{(2 n)}\left(x^{\mu}\right) \cos \frac{2 n y}{R}, \\
\phi_{+-}\left(x^{\mu}, y\right) & =\sqrt{\frac{2}{\pi R}} \sum_{n=0}^{\infty} \phi_{+-}^{(2 n+1)}\left(x^{\mu}\right) \cos \frac{(2 n+1) y}{R}, \\
\phi_{-+}\left(x^{\mu}, y\right) & =\sqrt{\frac{2}{\pi R}} \sum_{n=0}^{\infty} \phi_{-+}^{(2 n+1)}\left(x^{\mu}\right) \sin \frac{(2 n+1) y}{R} \\
\phi_{--}\left(x^{\mu}, y\right) & =\sqrt{\frac{2}{\pi R}} \sum_{n=0}^{\infty} \phi_{--}^{(2 n+2)}\left(x^{\mu}\right) \sin \frac{(2 n+2) y}{R} .
\end{aligned}
$$

The above Fourier decompositions lead to the following observations: Upon compactification, the fields $\phi_{++}^{(2 n)}$ acquire a mass $2 n / R$, while $\phi_{+-}^{(2 n+1)}$ and $\phi_{-+}^{(2 n+1)}$ acquire a mass $(2 n+1) / R$ and $\phi_{--}^{(2 n+2)}$ acquire a mass $(2 n+2) / R$. This implies that the only fields which can have massless components are $\phi_{++}^{(2 n)}$. The other interesting consequence is that only $\phi_{++}$and $\phi_{+-}$can have non-vanishing components on the $y=0$ brane. These simple observations have remarkable consequences. For example, consider the case when $\phi\left(x^{\mu}, y\right)$ transforms as a multiplet under some symmetry group G. Now if $P\left(\right.$ or $P^{\prime}$ ) are chosen to be different for different components of the multiplet, then upon compactification a symmetry reduction will result. The usual line of action is the following: Start with a 5-dimensional $N=2$ SUSY theory invariant under the gauge group SU(5); upon the first compactification by $Z_{2}$ the conjugated fields are projected out and the $N=2$ SUSY reduces to $N=1$ SUSY but still respecting the gauge $\mathrm{SU}(5)$, on the second compactification by $Z_{2}^{\prime}$ the $\mathrm{SU}(5)$ gauge symmetry is broken to the $\mathrm{SM}$ gauge group $\mathrm{SU}(3) \times \mathrm{SU}(2) \times \mathrm{U}(1)$ with a unbroken $N=1$ SUSY. The assignment of parities in the KAF model (which we briefly summarise here but for details refer the readers to the original references $[6,7])$ are such that only the $(+,+)$ fields, i.e. fields which have massless components and do not vanish on the $y=0$ brane (which is taken to be the 'visible' brane) are the gauge and Higgs multiplets of the MSSM. Other components like the coloured triplet Higgs fields, for example, do not have $(+,+)$ parity assignments and therefore do not have any massless component but acquire a minimum mass of order $1 / R \sim M_{\mathrm{GUT}}$. This provides a very elegant solution to the problem of doublet-triplet splitting and constitutes one of the attractive features of the orbifold compactification advocated in the KAF model. 
The 5-dimensional theory contains the vector bosons $A_{M}(M=(\mu, 5)$, where $\mu=0,1,2,3)$, two gauginos $\lambda, \lambda^{c}$ and a real scalar $\sigma$, all of them transforming in the adjoint representation of SU(5). This is equivalent to a $N=1$ vector multiplet $V\left(A_{\mu}, \lambda\right)$ and a chiral multiplet $\Sigma\left(\phi_{\Sigma}, \psi_{\Sigma}\right)$ in the adjoint representation, with $\phi_{\Sigma}=\left(\sigma+i A_{5}\right) / \sqrt{2}$ and $\psi_{\Sigma}=\lambda^{c}$. Then there are $N=1$ chiral Higgs multiplets $H_{5}$ and $H_{\overline{5}}$ and their conjugated partners. $H_{5}$ and $H_{\overline{5}}$ contain the scalar Higgs doublets $H_{u}^{D}$ and $H_{d}^{D}$ and the corresponding scalar triplets $H_{u}^{T}$ and $H_{d}^{T}$. Their $P$ and $P^{\prime}$ parity assignments are listed in Table 1 of [7]. We just mention that $A_{\mu}, \lambda, H_{u}^{D}$ and $H_{d}^{D}$ are $(+,+)$ fields, which have zero modes and also can have nonvanishing components at the branes.

For the quark and lepton matter, there is more freedom in the assignments of parities. First of all, we notice that $N=2$ SUSY does not allow any trilinear Yukawa interaction in the bulk. In $N=2$ SUSY, all interactions are gauge interactions. The Yukawa interactions can however be placed in the branes where $N=2$ SUSY breaks to $N=1$ SUSY. Now, as Altarelli and Feruglio [7] have argued, the matter fields are located only at the branes. They cannot propagate in the bulk, because if they do, it is not possible to construct a bulk interaction invariant under the gauge $\operatorname{SU}(5)$ and the $\left(Z_{2}, Z_{2}^{\prime}\right)$ parities. For the sake of illustration, let us denote the matter fields in the SU(5) representation as: $10 \equiv(Q, U, E)$ and $\overline{5} \equiv(L, D)$, which are $N=1$ chiral multiplets. Since all these matter fields can reside in the $y=0$ brane, the $Z_{2}$ parities for all of them are positive. What about the $Z_{2}^{\prime}$ parities of the matter fields? They have to be fixed from the gauge interaction of the matter fields with the gauge fields and the Yukawa interaction of the matter fields with the Higgs fields in 4 dimensions. In the following, let us briefly summarise how it is realised in the KAF model.

First we explicitly write down all the relevant interactions. For that we denote a matter chiral multiplet by $\Phi_{M} \equiv\left(\phi_{M}, \psi_{M}\right)$, where $M=(Q, L, U, D, E)$, with $\phi_{M}$ and $\psi_{M}$ being the scalar and fermionic components respectively. The gauge interaction induced at $y=0$ by the Kahler potential,

$$
K \equiv 10^{\dagger} e^{V} 10+\overline{5}^{\dagger} e^{V \overline{5}}
$$

when decomposed in components, reads:

$$
\begin{gathered}
\mathcal{L}_{g}=\mathcal{L}_{g}^{a}+\mathcal{L}_{g}^{\hat{a}}, \\
\mathcal{L}_{g}^{a}=\sum_{M} \overline{\psi_{M}} \bar{\sigma}^{\mu} T^{a} \psi_{M} A_{\mu}^{a}, \\
\mathcal{L}_{g}^{\hat{a}}=\overline{\psi_{Q}} \bar{\sigma}^{\mu} T^{\hat{a}} \psi_{U} A_{\mu}^{\hat{a}}+\overline{\psi_{Q}} \bar{\sigma}^{\mu} T^{\hat{a}} \psi_{E} A_{\mu}^{\hat{a}}+\overline{\psi_{L}} \bar{\sigma}^{\mu} T^{\hat{a}} \psi_{D} A_{\mu}^{\hat{a}}+\text { h.c. }
\end{gathered}
$$

In the above equations, $T^{a}$ and $T^{\hat{a}}$ correspond to the unbroken and broken $\mathrm{SU}(5)$ generators, respectively, and $\bar{\sigma}_{\mu}=\left(1,-\sigma^{k}\right)$ with $\sigma^{k}$ being the Pauli matrices. The effective 4-dimensional Lagrangian is given by:

$$
\mathcal{L}_{g}^{(4)}=\int d y[\delta(y)+\delta(-y+\pi R)] \mathcal{L}_{g}(y) .
$$

The Yukawa interaction on the branes is given by

$$
W^{(4)}=\int d y[\delta(y)+\delta(-y+\pi R)] W(y) \text {, where }
$$




$$
W(y)=y_{u} 1010 H_{5}+y_{d} 10 \overline{5} H_{\overline{5}}+y_{R} 10 \overline{5} \overline{5} .
$$

The $1010 H_{5}$ term contains the up quark mass term $Q U H_{u}^{D}, 10 \overline{5} H_{\overline{5}}$ contains the down quark and electron mass terms $Q D H_{d}^{D}$ and $L E H_{d}^{D}$ respectively, and $10 \overline{5} \overline{5}$ can be decomposed as $Q L D+L L E+$ $U D D$. Now comes the task of assigning the matter $P^{\prime}$ parities. The following three observations have been made in [7]:

1. $P^{\prime}(Q, L, U, D, E)=(+,+,+,+,+)$ : After $y$-integration in Eq. (7), the contribution from Eq. (5) survives and that from Eq. (6) vanishes. So the $\mathrm{SU}(5)$ gauge interaction is broken to the SM gauge interaction. But the simultaneous presence of the lepton and baryon number violating operators inside $10 \overline{5} \overline{5}$ mediates proton decay at a very rapid rate.

2. $P^{\prime}(Q, L, U, D, E)= \pm(+,+,-,-,-)$, or $\pm(+,-,-,+,-)$ : Full $\mathrm{SU}(5)$ symmetry is preserved on the branes. But, in the first case, the Yukawa interactions are $P^{\prime}$ odd and vanish after the $y$ integration, while in the second case, up-type quark masses cannot be generated (since $1010 H_{5}$ is $P^{\prime}$ odd) leading to a unrealistic picture.

3. $P^{\prime}(Q, L, U, D, E)=(+,-,+,+,-)$ : $\mathrm{SU}(5)$ invariance is lost. The interactions only respect $\mathrm{SU}(3)$ $\times \mathrm{SU}(2) \times \mathrm{U}(1)$. But (a) the necessary terms for yielding matter masses, namely, $Q U H_{u}^{D}+$ $Q D H_{d}^{D}+L E H_{d}^{D}$ can be generated, (b) the first term in Eq. (6), an essential ingredient for vector boson mediated proton decay, is prohibited, (c) coloured Higgsino mediated proton decay operators, contained in Eq. (9) but not explicitly shown here (see [7]), is again forbidden, and, finally, (d) the lepton number violating $L L E$ and $L Q D$ terms, inside $10 \overline{5} \overline{5}$, are also disallowed. Note, even though the baryon number violating $U D D$ operator is allowed, this alone, without the assistance of any lepton number violating operator, cannot drive proton decay. We also observe that with this choice of $P^{\prime}$ parities, the SU(5) invariance at the brane is abandoned, and the symmetry corresponds to that of the residual $\mathrm{SU}(3) \times \mathrm{SU}(2) \times \mathrm{U}(1)$. Still, some specific $\mathrm{SU}(5)$ properties, like $m_{b}=m_{\tau}$, can be realised.

So the lesson is that the most convenient $\left(P, P^{\prime}\right)$ assignments of matter fields are: $(Q, U, D)=$ $(+,+)$, and $(L, E)=(+,-)$. The 4-dimensional superpotential that follows this assignment is

$$
W^{(4)}=2 \int d y \delta(y)\left[y_{u} Q U H_{u}^{D}+y_{d}\left(Q D H_{d}^{D}+L E H_{d}^{D}\right)+y_{R} U D D\right] .
$$

The first three terms in Eq. (10) are responsible for the mass generation of up-quarks, down-quarks and charged leptons respectively. The last term is baryon number violating, and we will see how it provides a handle to test this model. Even though terms involving $H_{u}^{T}$ and $H_{d}^{T}$ arise separately (not explicitly shown in Eq. (10)), there is no bilinear Higgs triplet mixing term. In fact, with the parity assignments above, proton decay is forbidden at all orders.

We find it appropriate to mention here that the assignments of $P^{\prime}$ parities made in the KAF model are by no means the only possiblity. In the scenario presented by Hall and Nomura [9], the $P^{\prime}$ assignments correspond to the case (2) listed above. The reason that in [9] this choice works is that Hall and Nomura suggest that in some cases (e.g. $y_{u}$ ) the same Yukawa coupling at $y=0$ and $\pi R$ may have a relative sign. This may, indeed, be true if the Yukawa couplings were functions of the 5th co-ordinate $y$ and this latter circumstance can occur quite naturally in string theories where the couplings arise as vacuum expectation values of moduli. So if one were to start from string-inspired considerations, it may seem that the choice made by Hall and Nomura is justified. In the KAF model, 
the approach is to treat the couplings as independent of $y$, i.e. to have no relative sign between the same Yukawa coupling at $y=0$ and $\pi R$. On the other hand, the Hall-Nomura approach would allow a relative sign between the same Yukawa coupling at $y=0$ and $\pi R$, thus permitting the $P^{\prime}$ odd brane operators survive after the $y$ integration. This is precisely the point where Hall and Nomura [9] differ with Alterelli and Feruglio [7]. While in [9] a SU(5) symmetric brane interaction at $y=0$ could be written down, in [7] the symmetry on the $y=0$ brane is that of the residual $\mathrm{SU}(3) \times \mathrm{SU}(2) \times \mathrm{U}(1)$. Furthermore, the choice of $P^{\prime}$ parities in [9] predicts $A_{\mu}^{\hat{a}}$ boson exchanged proton decay at a rate to be seen in the forthcoming experiments, while the choice made in [7] prohibits all kinds of proton decay at all orders. The other important difference between [7] and [9] is that while the former allows baryon number violating term in the 4-dimensional superpotential, the latter contains $R$-parity as a discrete subgroup thus justifying the absence of either the lepton number or the baryon number violating interaction.

Thus having noted down the principal differences between [7] and [9], we now go back to the formalism of [7] which we have called the KAF model. Even though it is a GUT model, the conventional 'smoking gun' signal of proton decay is absent at all orders. Turning the argument around, if proton decay is discovered, this model will be ruled out. But then how we can experimentally verify this model? What are its observable consequences? We address ourselves to precisely this question in the present work. We observe that the baryon number violating $U D D$ term in Eq. (10) provides the clue as to where to look for its traces.

As such it is not possible to make any concrete estimation of the size of $y_{R}$ in the KAF model. The best we can do is to argue a purely geometrical suppresion of the $y_{u}$ or $y_{d}$ term, that contains one bulk field $\left(H_{u}^{D}\right.$ or $\left.H_{d}^{D}\right)$ and two brane-localised matter fields, as compared to the $y_{R}$ induced term which contains all three brane-localised fields. The basic point is that while writing down the 4-dimensional effective Lagrangian involving a 5-dimensional bulk field, the latter has to be Fourier decomposed into a tower of Kaluza-Klein modes with a normalisation $1 / \sqrt{l}$ due to compactification, where $l=\pi R / 2$. In fact each time a bulk field appears in a 4-dimensional effective Lagrangian, it is accompanied by a volume suppression factor $\epsilon \equiv 1 / \sqrt{M_{*} l}$, where $M_{*}$ is the UV cutoff scale for the effective theory which appears as a result of the correct normalization to provide the zero modes of the bulk field with appropriate 4-dimensional canonical dimensions. For the KAF model, this line of reasoning has the implication that the usual Yukawa couplings of the brane-localised matter fields with the bulk Higgs field are suppressed by $\mathcal{O}(\epsilon)$ at the GUT scale as compared to the baryon number violating Yukawa couplings which involve all three matter fields.

But in the absence of any flavour physics whatsoever on the brane in the KAF model it is not possible to make any numerical estimate of the size of this suppression. On the other hand, in order to admit any flavour physics on the brane, different matter fields will have to be allowed to propagate in different dimensions in the bulk. However, we stated in the beginning that bulk matter does not respect $\mathrm{SU}(5)$ gauge invariance in 5 dimensions. Still, as has been observed in Refs. [9, 10], one can order another set of $5^{\prime}$ and $10^{\prime}$ and their mirror partners to achieve a consistent bulk matter formalism. Although this amounts to a departure from the usual GUT structure, one can still ensure gauge coupling unification. In any case, this is the minimal alteration from the 'traditional line' needed to activate any flavour physics at all on the brane. Without going into the details of [10], we simply take a very crude but reasonable estimate the authors of [10] have made, namely, $\epsilon \sim 1 / 10$. Even though in the KAF model there is no such extra $5^{\prime}$ and $10^{\prime}$, the approximate size of the volume suppression that we borrow from [10] seems a reasonable one. This choice allows us to make a general statement that in the KAF model a generic baryon number violating coupling at $M_{\mathrm{GUT}}$ is about an order of 
magnitude larger than a generic Yukawa coupling at the same scale. In the following paragraph we try to make this statement more explicit.

Even though the KAF model does not discuss any flavour physics at all, we observe a significant hierarchy among the experimental quark and lepton masses at low energy anyway. To this end, we make the following simplistic assumption that the baryon number violating couplings may have a kind of generational hierarchy that is observed among the usual matter-Higgs Yukawa couplings. We first write down the effective 4-dimensional baryon number violating superpotential with explicit generation indices as $\lambda_{i j k}^{\prime \prime} U_{i} D_{j} D_{k}$ (with an antisymmetry between $j$ and $k$ ) and the 4-dimensional quark-Higgs superpotential as $h_{d}^{i j} Q_{i} H_{d} D_{j}+h_{u}^{i j} Q_{i} H_{u} U_{j}$, where $H_{u}$ and $H_{d}$ correspond to the zero modes of $H_{u}^{D}$ and $H_{d}^{D}$ respectively. For simplicity, we assume only two types of $\lambda^{\prime \prime}$ couplings, those involving the maximum and minimum possible values of $(i j k)$, with the following relation at the GUT scale:

$$
\lambda_{323}^{\prime \prime} \sim h_{t} / \epsilon, \quad \lambda_{112}^{\prime \prime} \sim h_{u} / \epsilon .
$$

As the experimental upper limits imply, the other $\lambda_{i j k}^{\prime \prime}$ couplings are presumably related to the light quark Yukawa couplings. These assumptions are admittedly simplistic, but we will try to see what these simple assumptions will lead us to. We make a remark here that if the $\lambda^{\prime \prime}$ couplings sit on their experimental upper limits, it turns out that $\lambda_{323}^{\prime \prime} \gg \lambda_{112}^{\prime \prime}[11]$.

With the kind of hierarchy at GUT scale given by Eq. (11), particularly with $\lambda_{323}^{\prime \prime}$ being an order of magnitude larger than $h_{t}$ and more specifically both being non-perturbative at the GUT scale, an interesting possibility arises when both are driven to their fixed point values at the electroweak scale. This has been considered in [12] (see also [13]), and the fixed point solutions are obtained in terms of the gauge couplings at the scale $M_{Z}$ as $\left(Y_{t}=h_{t}^{2} / 4 \pi, Y_{3}=\lambda_{323}^{\prime \prime 2} / 4 \pi\right)$ :

$$
Y_{t} \simeq \frac{1}{16}\left[8 \alpha_{3}+9 \alpha_{2}+\frac{9}{5} \alpha_{1}\right], Y_{3} \simeq \frac{1}{16}\left[\frac{56}{3} \alpha_{3}-3 \alpha_{2}+\frac{23}{15} \alpha_{1}\right]
$$

Using the values $\alpha_{3}\left(M_{Z}\right)=0.118, \alpha_{2}\left(M_{Z}\right)=0.0336$ and $\alpha_{1}\left(M_{Z}\right)=0.0167$, we obtain $m_{t}=174 \mathrm{GeV}$ (for $\tan \beta>5$ ) and $\lambda_{323}^{\prime \prime}=1.3$ at the weak scale.

What is the impact of such a large $\lambda_{323}^{\prime \prime}$ on electroweak observables? Certainly, this coupling will contribute to $Z \rightarrow b \bar{b}$ and $s \bar{s}$ via quark-squark triangle graphs [14]. The $Z$ coupling to $q_{R}(q=s, b)$ will be modified by an amount $\lambda_{323}^{\prime \prime 2} N_{c} f\left(m_{t}^{2} / \tilde{m}^{2}\right) / 16 \pi^{2}$, where $N_{c}=3$ and $\tilde{m}$ is an average squark mass. The explicit expression of the function $f$ in terms of the Passarino-Veltman two- $(B)$ and three$(C)$ point functions are given in [14]. The best constraint on $\lambda_{323}^{\prime \prime}$ comes from $R_{l}=\Gamma_{\text {had }} / \Gamma_{l}$ (where $\left.R_{l}^{\mathrm{SM}}=20.740, R_{l}^{\text {exp }}=20.767 \pm 0.025\right)$, given by $\lambda_{323}^{\prime \prime} \leq 0.83($ at $2 \sigma)$ for a common squark mass of $100 \mathrm{GeV}$. We have used the latest LEP Electroweak Working Group report [15] to obtain the above limit as an update of our previous work [14]. This implies that the fixed point value $\lambda_{323}^{\prime \prime} \simeq 1.3$ can be accommodated by taking the squark mass near $150 \mathrm{GeV}$ or so. We make a remark in passing that with this interaction it is difficult to sizably alter the forward-backward asymmetry in the $b$ quark channel keeping consistency with the hadronic width measurement at the same time.

Another probe of $\lambda_{323}^{\prime \prime}$ is the $t \bar{t}$ production at the Tevatron [16]. In the presence of the baryon number violating couplings, in addition to the SM process, $q \bar{q} \rightarrow t \bar{t}$ mediated by $s$-channel gluon exchange, there are new $t$-channel processes involving the exchange of a squark. This new process can lead to a significant enhancement of the $t \bar{t}$ cross-section. This enhancement can be effectively probed in Run II of the Tevatron because of the high-statistics that will be achieved in Run II and 
also because $t \bar{t}$ production at the Tevatron energy is dominated by $q \bar{q}$-initiated channel which will be affected by the baryon number violating operators. With a value of $\lambda_{323}^{\prime \prime} \sim 1.3$ as suggested by the above arguments, we compute the integrated $t \bar{t}$ production cross-section for $\sqrt{s}=2 \mathrm{TeV}$. The SM value for the $t \bar{t}$ cross-section at this energy is about $9.4 \mathrm{pb}$ and assuming an integrated luminosity of $2 \mathrm{fb}^{-1}$ we expect about 18,500 SM events. Assuming purely statistical errors, we then estimate the sensitivity of this process to probe the baryon number violating channel. We find that squark masses to values somewhat larger than $300 \mathrm{GeV}$ at the $95 \%$ C.L. level can be probed for $\lambda_{323}^{\prime \prime} \sim 1$.

What about the $\lambda^{\prime \prime}$ couplings which involve lower generation indices? Intuitively, if our approximation in Eq. (11) is sensible, then these couplings will be an order of magnitude larger than the lower generation quark Yukawa couplings. In view of the hierarchies among quark masses, it is rather impossible to ascertain any value for those $\lambda^{\prime \prime}$ couplings, but roughly we can expect them to be in the range $10^{-5}-10^{-3}$ at the GUT scale and more or less in the same range at the weak scale. Some of these couplings will induce large neutron-antineutron $(n-\bar{n})$ oscillation or drive double nucleon decay into two kaons at a rapid rate. The existing bounds from $n-\bar{n}$ oscillation are:

$$
\lambda_{113}^{\prime \prime} \leq 5 \times 10^{-3}, \lambda_{312}^{\prime \prime} \leq 1.5 \times 10^{-2}, \lambda_{313}^{\prime \prime} \leq 2.0 \times 10^{-2},
$$

for an average squark mass of $200 \mathrm{GeV}$. These bounds have been obtained [17, 18] using the $n-\bar{n}$ oscillation time $\tau>1.2 \times 10^{8} \mathrm{~s}$ : there are some uncertainties though coming from the nuclear matrix element calculations. The bound that follows from the consideration of $N N \rightarrow K K$ is [17, 18]:

$$
\lambda_{112}^{\prime \prime} \leq 1.0 \times 10^{-6},
$$

again for a squark mass around $200 \mathrm{GeV}$. This bound also depends on a suitable choice of the ratio between the hadronic scale and the SUSY breaking scale. The values of $\lambda^{\prime \prime}$ couplings close to their experimental upper limits can be easily accommodated in the KAF model. Turning the argument around, if we observe a significant enhancement (compared to the SM) in those channels, this might be interpreted in terms of the $U D D$ couplings. The other lesson we learn is that if we attempt to construct a realistic flavour model, apart from the $\lambda_{323}^{\prime \prime}$, all other $\lambda^{\prime \prime}$ couplings would have to be small (see the bounds listed in [11]).

In conclusion, the simplest version of the supersymmetric SU(5) GUT models with an extra dimension compactified on a $S^{1} /\left(Z_{2} \times Z_{2}^{\prime}\right)$ orbifold, as introduced by Kawamura and further developed by Altarelli and Feruglio, solves the doublet-triplet splitting problem and suppresses proton decay at all orders by a suitable implementation of matter $Z_{2}$ and $Z_{2}^{\prime}$ parities. Since proton decay constitutes the 'smoking gun' signal of the Grand Unified models, a question that naturally arises is how we experimentally verify this particular incarnation of Grand Unification. Interestingly, this model admits baryon number violating couplings, and in this paper we seek to exploit the effects of these couplings on neutron-antineutron oscillations, double nucleon decay into two kaons, hadronic decay widths of the $Z$ boson, and $t \bar{t}$ production cross-section in Run II of the Tevatron. Although a significant deviation from the SM expectations on these observables will strengthen the case for a baryon number violating interaction, any future observation of proton decay will suffice to rule out the KAF model.

Acknowledgements: It is a pleasure to thank G. Altarelli, F. Feruglio, J. March-Russell and Y. Nomura for useful discussions. GB thanks the Theory Division, CERN, Geneva, and ICTP, Trieste, for hospitality during the period in which most of the work has been done. 


\section{References}

[1] S. Dimopoulos, H. Georgi, Nucl. Phys. B 193 (1981) 150; N. Sakai, Z. Phys. C 11 (1981) 153; R.K. Kaul, Phys. Lett. 109 B (1982) 19; R.K. Kaul, P. Majumdar, Nucl. Phys. B 199 (1982) 36.

[2] J. Ellis, D.V. Nanopoulos, S. Rudaz, Nucl. Phys. B 202 (1982) 43; P. Nath, A.H. Chamseddine, R. Arnowitt, Phys. Rev. D 32 (1985) 2348.

[3] J. Ellis, S. Kelley, D.V. Nanopoulos, Phys. Lett. B 260 (1991) 131; U. Amaldi, W. de Boer, H. Furstenau, Phys. Lett. B 260 (1991) 447.

[4] H. Murayama, A. Pierce, Phys. Rev. D 65 (2002) 055009 [hep-ph/0108104]; G. Altarelli, F. Feruglio, I. Masina, JHEP 0011 (2000) 040 [hep-ph/0007254].

[5] Y. Hayato et al. [Super-Kamiokande Collaboration], Phys. Rev. Lett. 83 (1999) 1529 [hepex/9904020].

[6] Y. Kawamura, Prog. Theor. Phys. 105 (2001) 999 [hep-ph/0012125].

[7] G. Altarelli, F. Feruglio, Phys. Lett. B 511 (2001) 257 [hep-ph/0102301].

[8] A.B. Kobakhidze, Phys. Lett. B 514 (2001) 131 [hep-ph/0102323].

[9] L. Hall, Y. Nomura, Phys. Rev D 64 (2001) 055003 [hep-ph/0103125].

[10] L. Hall, J. March-Russell, T. Okui, D. Smith, hep-ph/0108161. See also, A. Hebecker, J. MarchRussell, Nucl. Phys. B 613 (2001) 3 [hep-ph/0106166]; A. Hebecker, J. March-Russell, hep$\mathrm{ph} / 0107039$.

[11] G. Bhattacharyya, Nucl. Phys. Proc. Suppl. 52A (1997) 83 [hep-ph/9608415]; hep-ph/9709395; H. Dreiner, hep-ph/9707435; B.C. Allanach, A. Dedes, H. Dreiner, Phys. Rev. D 60 (1999) 075014 [hep-ph/9906209].

[12] V. Barger, M.S. Berger, R.J.N. Phillips, T. Wohrmann, Phys. Rev. D 53 (1996) 6407 [hepph/9511473].

[13] B. de Carlos, P.L. White, Phys. Rev. D 54 (1996) 3427 [hep-ph/9602381]; B. Brahmachari, P. Roy, Phys. Rev. D 50 (1994) 39, Erratum-ibid. D 51 (1995) 3974.

[14] G. Bhattacharyya, D. Choudhury, K. Sridhar, Phys. Lett. B 355 (1995) 193 [hep-ph/9504314].

[15] LEP Electroweak Working Group Report, LEPEWWG Note 2001-01: Status of winter 2001.

[16] D. Ghosh, S. Raychaudhuri, K. Sridhar, Phys. Lett. B 396 (1997) 177 [hep-ph/9608352].

[17] J.L. Goity, M. Sher, Phys. Lett. B 346 (1995) 69, Erratum-ibid. B 385 (1996) 500 [hep-ph/9412208]. For a more recent analysis, see K.S. Babu, R.N. Mohapatra, Phys. Lett. B 518 (2001) 269 [hepph/0108089].

[18] D. Chang. W.-Y. Keung, Phys. Lett. B 389 (1996) 294 [hep-ph/9608313]. 